
\title{
Mapeamento dos diagnósticos de enfermagem em uma
unidade de pneumologia*
}

\author{
Mapping nursing diagnoses in a pulmonology unit \\ Cuadro explicativo de los diagnósticos de enfermería en una unidad de neumología
}

\section{RESUMO}

Objetivos: Identificar os diagnósticos de enfermagem mais frequentes, segundo a taxonomia II da NANDA - I; as características definidoras, fatores relacionados e de risco que deram suporte para elaboração dos mesmos, em pacientes com distúrbios respiratórios. Métodos: Estudo transversal, realizado em um hospital universitário da cidade de São Paulo. Os diagnósticos de enfermagem foram elaborados pela pesquisadora e submetido à validação consensual de três especialistas. Os resultados foram descritos baseados na frequência absoluta e percentual. Resultados: Trinta e quatro pacientes foram avaliados e 35 diagnósticos de enfermagem apresentaram concordância entre a pesquisadora e os especialistas. Conclusão: Estudos epidemiológicos que informam sobre a frequência dos diagnósticos de enfermagem em uma população contribuem para organizar a base de conhecimento de enfermagem e prever os cuidados de enfermagem necessários a uma população específica.

Descritores: Sistema respiratório; Diagnóstico de enfermagem; Saúde do adulto

\begin{abstract}
Objectives: To identify the most frequent nursing diagnoses according to NANDA International's Taxonomy II; the defining characteristics, related factors and risk factors, in patients with respiratory disorders. Methods: Cross-sectional study in a university hospital in São Paulo. The nursing diagnoses were determined by the researcher, and subjected to consensual validation by three experts. Results are based on absolute frequency and percentage. Results: Thirty-four patients were evaluated and 35 nursing diagnoses were identified that achieved consensus across the researcher and the experts. Conclusion: Epidemiological studies that report on the frequency of nursing diagnoses in a population contribute to organizing the knowledge base of nursing and the appropriate provision of nursing care required by a specific population.
\end{abstract}

Keywords: Respiratory system; Nursing diagnoses; Adult health

\section{RESUMEN}

Objetivos: Identificar los diagnósticos de enfermería más frecuentes, según la taxonomia II de la NANDA - I; las características definidoras, factores relacionados y de riesgo que dieron soporte a la elaboración de los mismos, en pacientes con disturbios respiratorios. Métodos: Estudio transversal, realizado en un hospital universitario de la ciudad de Sao Paulo. Los diagnósticos de enfermería fueron elaborados por la investigadora y sometido a la validación consensual de tres especialistas. Los resultados fueron descritos basados en la frecuencia absoluta y porcentual. Resultados: Fueron evaluados treinta y cuatro pacientes presentando concordancia entre la investigadora y los especialistas 35 diagnósticos de enfermería. Conclusión: Estudios epidemiológicos que informan sobre la frecuencia de los diagnósticos de enfermería en una población, contribuyen para organizar la base del conocimiento de enfermería y prever los cuidados de enfermería necesarios a una población específica.

Descriptores: Sistema respiratorio; Diagnóstico de enfermería; Salud de los adultos

\footnotetext{
* Trabalho realizado na Unidade de Pneumologia, Hospital São Paulo - HSP - São Paulo (SP), Brasil.

${ }^{1}$ Enfermeira da Unidade de Obstetrícia do Hospital São Paulo - HSP - São Paulo (SP), Brasil.

${ }^{2}$ Doutora em Enfermagem, Gerente de Enfermagem das Clinicas Medicas Especializadas do Hospital São Paulo - São Paulo (SP), Brasil.

${ }^{3}$ Especialista em Enfermagem em Pneumologia - modalidade residência - São Paulo (SP), Brasil.

${ }^{4}$ Especialista em Enfermagem Clinica e Cirúrgica e Enfermeiro Encarregado da Unidade de Pneumologia do Hospital São Paulo - São Paulo (SP), Brasil.

${ }^{5}$ Doutora em Ciências.Professor Adjunto da Disciplina de Fundamentos de Enfermagem e Enfermagem Médico Cirúrgica da Escola Paulista de Enfermagem,

Universidade Federal de São Paulo - UNIFESP - São Paulo (SP), Brasil.
} 


\section{INTRODUÇÃO}

O sistema respiratório, em particular, está exposto às agressões do ambiente e, frequentemente, é sede de alterações de maior ou menor intensidade e gravidade.

$\mathrm{O}$ alto índice de mortalidade por doenças do trato respiratório é um grande problema de saúde pública. Estatísticas divulgadas pelo DataSus Censo-2006 revelam que doenças do sistema respiratório estão em terceira posição no ranking de maiores internações hospitalares ${ }^{(1)}$.

As projeções de prevalência das doenças pulmonares mostram um aumento nas próximas décadas em razão de contínua exposição aos fatores de risco, sobretudo, a poluição e o tabagismo, e o envelhecimento da população mundial.

No inverno, a prevalência aumenta, envolvendo as vias aéreas inferiores como as superiores ${ }^{(2-4)}$.

O processo de enfermagem é entendido como uma atividade intelectual, premeditada e deliberada, por meio da qual a prática de enfermagem é dirigida dentro de uma ordem e de um modo sistemático, no qual o DE é uma fase desse processo ${ }^{(5-9)}$.

Para elaborar o DE, é necessário a coleta de dados, o exame físico e o raciocínio clínico atencioso com base técnico-científica na anatomia, fisiologia, fisiopatologia e grande habilidade com a Sistematização da Assistência de Enfermagem (SAE) e com um sistema de classificação de diagnósticos ${ }^{(7)}$.

O processo torna-se fundamental em pacientes portadores de doença do trato respiratório, visto que esta afecção é extremamente dinâmica e necessita de constante avaliação e replanejamento da assistência.

A situação clínica do doente, a natureza das informações que se quer obter e as habilidades cognitivas e perceptivas da enfermeira são fatores que influenciam a organização de modelos para a coleta dos dados. Quando a assistência se dá em unidades especializadas, associa-se maior probabilidade de ocorrência e de identificação de determinadas necessidades de cuidados. Em pacientes que compartilham problemas de saúde semelhantes, a maior probabilidade de ocorrência de certas necessidades de cuidados deve ser usada para nortear o conteúdo da coleta de informações. No entanto, é preciso ter cautela para que isso não se torne fator limitante para a identificação de necessidades pouco frequentes na população em questão.

As principais queixas relacionadas ao sistema respiratório são: a dispneia, a tosse, a expectoração, a hemoptise, a dor torácica e a rouquidão. No exame físico, podemos encontrar: cianose, baqueteamento digital, alterações da caixa torácica, do padrão respiratório, da expansibilidade, do frêmito toracovocal e dos sons à percussão e ausculta ${ }^{(10-11)}$. Quanto aos exames laboratoriais, os achados mais frequentes são alterações dos gases sanguíneos arteriais e hematócrito. Tais características compõem os indicadores clínicos dos DE relacionados ao sistema respiratório cujos fatores podem ser ambientais ou fisiológicos. As alterações de sinais e sintomas isolados ou agrupados exigem monitoração constante dos enfermeiros para elaboração das ações de enfermagem direcionadas às particularidades desta população.

Estudos epidemiológicos que informam sobre a frequência dos diagnósticos de enfermagem em uma população contribuem para organizar a base de conhecimento de enfermagem, prever os cuidados de enfermagem necessários a uma população específica e orientar a seleção de elementos no serviço e nos programas de educação ${ }^{(12)}$.

Baseado no exposto, os enfermeiros da unidade de Pneumologia ficaram estimulados para realizar pesquisa sobre esta etapa do processo de enfermagem para melhor compreensão das características definidoras e fatores relacionados que deram suporte à elaboração dos mesmos.

Assim, o objetivo do estudo foiIdentificar os diagnósticos de enfermagem mais frequentes, segundo a taxonomia II da NANDA - I em pacientes portadores de distúrbios respiratórios; e verificar quais características definidoras, fatores relacionados e fatores de risco compuseram e indicaram DE de clientes com distúrbios respiratórios.

\section{MÉTODOS}

Este trabalho foi autorizado pelo Comitê de Ética e Pesquisa sob o número CEP 0208/08. Trata-se de um estudo descritivo, transversal, realizado na unidade Pneumologia de um hospital universitário da cidade de São Paulo, SP.

Foram coletados os dados de pacientes que internaram no período da realização deste estudo e que consentiram participar assinando o Termo de Consentimento Livre e Esclarecido.

O levantamento dos dados foi realizado pela investigadora com o preenchimento do histórico de enfermagem utilizado na unidade no momento da internação do paciente. Após a obtenção destes dados, realizou-se o julgamento clínico para elaboração dos DE, com base nas características definidoras, dos fatores de risco e fatores relacionados presentes.

A coleta de dados contendo os dados referentes à entrevista, exame físico e dados laboratoriais foi encaminhada a três enfermeiros especialistas para validação dos diagnósticos de enfermagem propostos. Eles indicaram no instrumento elaborado pela investigadora, os diagnósticos de enfermagem, características definidoras e fatores relacionados que julgaram pertinentes a cada paciente. Assim, obtivemos, para cada paciente, quatro relações de diagnósticos (uma da pesquisadora principal e três dos especialistas). Estes enfermeiros possuíam no mínimo pósgraduação latu sensu, experiência de pelo menos 1 ano na prática de sistematização da assistência de enfermagem e na área de pneumologia, ou participação em pesquisa de identificação de diagnósticos de enfermagem. 
A investigadora confrontou as quatro relações de diagnósticos de enfermagem obtidas. Foram considerados DE validados para o estudo, os que tiveram concordância de, pelo menos, três dos envolvidos.

A seguir, foi realizada uma análise para verificar quais foram os DE mais frequentes e com base nestes, as características definidoras e os fatores de risco os compuseram.

\section{RESULTADOS}

Tabela 1 - Diagnósticos de Enfermagem identificados nos pacientes internados na Unidade de Pneumologia. São Paulo, 2008.

\begin{tabular}{|c|c|}
\hline Diagnósticos de Enfermagem & $\mathbf{n}$ \\
\hline Troca de gases prejudicada & 27 \\
\hline Privação de sono & 20 \\
\hline Risco de queda & 20 \\
\hline $\begin{array}{l}\text { Nutrição des equilibrada: menos do que as } \\
\text { necessidades cor porais }\end{array}$ & 15 \\
\hline Risco de infecção & 14 \\
\hline Dor aguda & 13 \\
\hline Fadiga & 13 \\
\hline Padrão respiratório ineficaz & 10 \\
\hline Ansiedade & 8 \\
\hline Tris teza crônica & 7 \\
\hline Deambulação prejudicada & 6 \\
\hline Ventilação espontânea prejudicada & 6 \\
\hline Comunicação verbal prejudicada & 5 \\
\hline $\begin{array}{l}\text { Déficit no autocuidado alimentar-se/ } \\
\text { banho higiene/ vestir-se arrumar }\end{array}$ & 5 \\
\hline Desobstrução ineficaz das vias aéreas & 5 \\
\hline Débito cardíaco diminuído & 4 \\
\hline Eliminação urinária prejudicada & 4 \\
\hline Perfusão tis sular ineficaz: cardiopulmonar & 4 \\
\hline Constipação & 3 \\
\hline Dor crônica & 3 \\
\hline Integridade da pele prejudicada & 3 \\
\hline Mobilidade física prejudicada & 3 \\
\hline Risco de aspiração & 3 \\
\hline Confusão aguda & 3 \\
\hline Deglutição prejudicada & 2 \\
\hline Incontinência urinária de urgência & 2 \\
\hline Memória prejudicada & 2 \\
\hline Volume excessivo de líquidos & 2 \\
\hline Distúrbio da imagem corporal & 2 \\
\hline Hipertermia & 1 \\
\hline Incontinência urinária funcional & 1 \\
\hline Intolerância à atividade & 1 \\
\hline $\begin{array}{l}\text { Nutrição des equilibrada: mai s do que as } \\
\text { necessidades cor porais }\end{array}$ & 1 \\
\hline Risco de constipação & 1 \\
\hline Adaptação prejudicada & 1 \\
\hline
\end{tabular}

Participaram do estudo 34 pacientes, com predomínio do sexo masculino $(59 \%)$ e a idade de 65 anos ou mais (41\%), sendo a idade média de 58 anos. Quanto ao tabagismo, 65\% dos pacientes possuíam o hábito de fumar em algum período da vida. Com o acréscimo dos clientes que tiveram contato com o tabaco por um longo período de tempo, unindo assim, os tabagistas e os passivos, encontramos na amostra $77 \%$ que ficaram expostos à poluição ambiental. Os diagnósticos médicos encontrados com maior frequência foram: Doença Pulmonar Obstrutiva Crônica (DPOC) (26\%), Neoplasia (23\%) e Pneumonia (18\%).

$\mathrm{Na}$ Tabela 1, estão apresentados os DE identificados nos pacientes portadores de distúrbios pulmonares.

Tabela 2 - Características Definidoras e Fatores Relacionados presentes nos Diagnósticos de Enfermagem identificados nos pacientes internados na Unidade de Pneumologia. São Paulo, 2008.

\begin{tabular}{|c|c|c|}
\hline $\begin{array}{l}\text { Diagnóstico de enfermagem: Troca de gases } \\
\text { p rejudicada }\end{array}$ & $\mathrm{n}$ & $\%$ \\
\hline \multicolumn{3}{|l|}{ Características definidoras } \\
\hline Disp neia & 24 & 89 \\
\hline Gases sanguíneo s arteriais anormais & 19 & 70 \\
\hline $\begin{array}{l}\text { Respira ção anormal (frequên cia, ritmo, } \\
\text { p rofundidade) }\end{array}$ & 16 & 59 \\
\hline Taquic ardia & 9 & 33 \\
\hline \multicolumn{3}{|l|}{ Fatores relacionados } \\
\hline D esequilíbrio n a ventila ção-perfusão & 27 & 100 \\
\hline Muda nças da membrana alveolocapilar & 24 & 89 \\
\hline Diagnóstico de en fermag em: Priva ção de sono & $\mathrm{n}$ & $\%$ \\
\hline \multicolumn{3}{|l|}{ Características definidoras } \\
\hline Mal-estar & 15 & 75 \\
\hline Transtornos perceptivos de sensação & 13 & 65 \\
\hline corporal perturbada & & \\
\hline Agitação & 12 & 60 \\
\hline Cansaço & 11 & 55 \\
\hline Sensibilida de aumentad a à dor & 10 & 50 \\
\hline Irritabilidade & 5 & 25 \\
\hline \multicolumn{3}{|l|}{ Fatores relacionados } \\
\hline D esconforto físico prolongado & 19 & 95 \\
\hline D esconforto p sicológico prolongado & 9 & 45 \\
\hline Diagnóstico de enfermag em: Fadiga & $\mathrm{n}$ & $\%$ \\
\hline \multicolumn{3}{|l|}{ Características de finidoras } \\
\hline Cansaço & 11 & 85 \\
\hline Verbalização de uma constante falta de energia & 6 & 46 \\
\hline Aumento das queixas físicas & 5 & 38 \\
\hline Falta de en ergia & 5 & 38 \\
\hline Incapacidade de manter as rotinas habituais & 5 & 38 \\
\hline \multicolumn{3}{|l|}{ Fatores relacionados } \\
\hline Ansiedade & 10 & 77 \\
\hline Estados de doença & 9 & 69 \\
\hline D epressão & 6 & 46 \\
\hline Estresse & 4 & 31 \\
\hline Diagnóstico de enfermagem: $\mathrm{R}$ isco de quedas & $\mathrm{n}$ & $\%$ \\
\hline \multicolumn{3}{|l|}{ Fatore s de risco } \\
\hline Força diminuída nas extremidades inferiores & 12 & 60 \\
\hline Idade acima de 65 anos & 11 & 55 \\
\hline Mobilidade fisica prejudicada & 7 & 35 \\
\hline Dificuldad es visua is & 6 & 30 \\
\hline Presença de doença aguda & 6 & 30 \\
\hline Neoplasias & 5 & 25 \\
\hline
\end{tabular}

Continua.. 
... continuação

\begin{tabular}{|c|c|c|}
\hline Diagnóstico de enfermag em: R isco de Infec ção & $\mathrm{n}$ & $\%$ \\
\hline \multicolumn{3}{|l|}{ Fatores de risco } \\
\hline Proced im entos inva sivos & 14 & 100 \\
\hline Defesas primárias inadequadas & 8 & 57 \\
\hline Defesas secundárias inadequadas & 6 & 43 \\
\hline D esnutrição & 4 & 29 \\
\hline \multirow{2}{*}{\multicolumn{3}{|c|}{$\begin{array}{l}\text { Diagnóstico de enfermagem: Nutrição } \\
\text { Desequilibrada: menos do que as necessidades } \\
\text { corporais }\end{array}$}} \\
\hline & & \\
\hline \multicolumn{3}{|l|}{ Características de finidoras } \\
\hline Falta de interesse na comida & 12 & 80 \\
\hline Perda de peso com ingestão adequa da de comida & 11 & 73 \\
\hline Aversão ao a to de comer & 6 & 40 \\
\hline Peso corporal $20 \%$ ou ma is a baixo do ideal & 6 & 40 \\
\hline \multicolumn{3}{|l|}{ Fatores relaciona dos } \\
\hline Fatores biológicos & 13 & 87 \\
\hline Incapacidade de ingerir os alimentos & 10 & 67 \\
\hline Fatores psicológicos & 8 & 53 \\
\hline Diagnóstico de enfermagem: D or Aguda & $\mathrm{n}$ & $\%$ \\
\hline \multicolumn{3}{|l|}{ Características de finidoras } \\
\hline Relato verbal de dor & 13 & 100 \\
\hline Expressão fa cial & 6 & 46 \\
\hline Distúrb io do sono & 4 & 31 \\
\hline Fatores relacionados & $\mathrm{n}$ & $\%$ \\
\hline Agente biológico lesivo & 10 & 77 \\
\hline Agente físico lesivo & 8 & 62 \\
\hline
\end{tabular}

Como pode ser visto nesta tabela, foram identificados 35 diagnósticos de enfermagem, e os apresentados em destaque (7) representam uma frequência de no mínimo $38 \%$.

Nos dados da Tabela 2, estão as Características Definidoras e os Fatores Relacionados ou de Risco que subsidiaram a elaboração dos sete DE.

\section{DISCUSSÃO}

De acordo com o DataSus Censo 2006, a faixa etária em idades acima de 65 anos representa $12,5 \%$ das internações hospitalares por doença do trato respiratório e $7,5 \%$ entre 50 e $64 \operatorname{anos}^{(1)}$. No presente estudo, podemos observar que a maioria dos pacientes era do sexo masculino $(59 \%)$ e a idade média foi de 58 anos, sendo a faixa etária de 50 a 64 anos que representou $32 \%$ e acima de 65 anos, $41 \%$,ou seja, bem maior do que a referida no Censo de 2006.

Em relação às doenças crônicas pulmonares distribuídas por sexo e idade, temos como parâmetro o inquérito domiciliar de saúde no Município de São Paulo no ano de 2003 que abrangeu população de quatro áreas do Estado de São Paulo (Campinas, Botucatu e regiões metropolitanas Taboão da Serra, Embu, Itapecerica e distrito administrativo do Butantã), com uma amostra de 3,360 indivíduos e demonstrou que a maior prevalência de DPOC está na faixa etária de 60 anos ou mais e no sexo masculino $(82,2 \%)^{(13)}$, o que está de acordo com os dados encontrados em nosso estudo.

Fumar é um hábito que atinge $65 \%$ dos pacientes da amostra, e unindo os tabagistas e os passivos, $77 \%$ têm relação com esta poluição ambiental. Salientamos a proporção da amostra deste estudo em relação ao tabagismo, dentro de uma unidade de pneumologia mesmo incluindo os de uso passivo, que estão expostos ao mesmo poluente.

O novo DE Contaminação tem como definição "Exposição a contaminantes ambientais em doses suficientes para causar efeitos adversos à saúde" e tem como característica definidora: "efeitos pulmonares da exposição à poluição pelo agente tabaco"(14). Não foi mencionado por nenhum dos enfermeiros especialistas, apenas pela investigadora, isto pode ter ocorrido pelo fato da investigadora ser estudante do curso de graduação, o que lhe confere um maior contato com os novos DE o que não ocorre com os enfermeiros. Este fato indica a necessidade de atualização por meio de pesquisa ou educação continuada ${ }^{(15)}$.

Em São Paulo, a prevalência de DPOC varia de 6\% a $15,8 \%$ em pacientes com idade igual ou superior a 40 anos $^{(16)}$. Em nosso estudo, encontramos $26 \%$ da amostra que possuiam o diagnóstico de DPOC.

A incidência do câncer de pulmão vem aumentando, aproximadamente, $3 \%$ a cada ano, apesar dos novos avanços tecnológicos alcançados nos últimos anos, tanto no diagnóstico como no tratamento ${ }^{(17)}$. Em nosso estudo, encontramos $23 \%$ dos pacientes com diagnóstico de neoplasia pulmonar.

A pneumonia é a primeira queixa nas consultas médicas em pacientes menores de 5 anos e maiores de 65 anos. A morbidade e mortalidade das pneumonias é influenciada pela presença de doenças associadas e idade acima de 65 anos $^{(18)}$. Os dados deste estudo indicam que $18 \%$ dos pacientes com diagnóstico de pneumonia.

Existem poucos artigos sobre DE relacionados ao sistema respiratório, sendo a maioria sobre o DE Desobstrução Ineficaz de Vias Aéreas ${ }^{(19-22)}$ que foram realizados em Unidades de Terapia Intensiva.

O estudo de um grupo de clientes com características em comum proporcionará a identificação dos diagnósticos de enfermagem, suas causas e expressões. Além disso, irá favorecer o direcionamento das intervenções de enfermagem e a identificação de estratégias para elevar a qualidade da assistência ${ }^{(23)}$.

O diagnóstico de enfermagem Troca de Gases Prejudicada apresentou a maior frequência na amostra estudada $(79 \%)$. Foi evidenciado pela anamnese revelando dispneia aos pequenos esforços (89\%), pelos exames laboratoriais que mostraram os gases sanguíneos arteriais fora dos níveis normais $(70 \%)$ e pelo exame físico constatando respiração anormal por alterações na frequência, ritmo ou amplitude em $59 \%$ dos pacientes e 
taquicardia em 33\%. Os fatores relacionados que deram suporte para o DE Troca de Gases Prejudicadas encontrados em nosso estudo, foram: desequilíbrio na ventilação perfusão $(100 \%)$ e mudanças na membrana alveolocapilar $(89 \%)$.

Estudo realizado com pacientes submetidos à ventilação mecânica, que pesquisou os sinais e sintomas dos diagnósticos de enfermagem relacionados ao sistema, respiratório, evidenciou que a principal característica definidora do diagnóstico de enfermagem troca de gases prejudicada foi gases sanguíneos arteriais anormais, considerado um sinal suficiente para identificar este diagnóstico, pois esta característica definidora estava presente em todos os pacientes ${ }^{(24)}$.

O DE Privação de Sono esteve presente em 59\% dos pacientes. As principais características definidoras que indicaram para este diagnóstico foram: a queixa de mal-estar em razão da dispneia posicional aliviada pela ortopneia $(75 \%)$ e transtornos perceptivos de sensação corporal perturbada, tais como dificuldades para adormecer e sono interrompido por meio de alterações sensoriais internas (estresse psicológico, dores e dispneia) $(65 \%)$.

Estudo semelhante com pacientes portadores de alterações hematológicas mostrou que o distúrbio no padrão do sono foi evidenciado pelas alterações físicas e comportamentais em relação ao estado de saúde. Dentre as comportamentais, estão: ansiedade, depressão, estresse, inatividade e outras ${ }^{(25)}$. Em nosso estudo, as alterações comportamentais encontradas foram: agitação $(60 \%)$ e irritabilidade (25\%) que não foram mencionadas na pesquisa anterior.

Outro fator importante que altera a qualidade do sono é a dor. A dificuldade de adormecer ocasionado pela sensibilidade aumentada pela dor foi relatada por $50 \%$ dos pacientes. A adaptabilidade à dor e o reconhecimento da influência de atitudes e comportamentos na expressão da dor é uma ideologia organizada pelos centros multidisciplinares de dor ${ }^{(26)}$. Estar livre da dor produz uma sensação de intenso conforto e bem-estar, contribuindo para a melhora da a qualidade do sono. Outro DE que possui grande relevância é o Risco de Quedas, presente em 59\% dos pacientes. Os principais fatores de risco que validaram este diagnóstico foram: força diminuída nas extremidades inferiores (60\%) e idade acima de 65 anos (55\%). Estudo realizado avaliando a correlação entre risco de queda e idade mostrou que pessoas acima de 60 anos tornamse mais propensas a quedas pelas fortes mudanças na instabilidade postural, mudanças no passo, redução de movimentos, mudanças na musculatura, articulações enrijecidas e redução de ossos saudáveis ${ }^{(27)}$.Estes dados corroboram com os encontrados em nosso estudo, no qual a maioria dos pacientes tinha idade superior a 60 anos.

O DE Nutrição desequilibrada: menos do que as necessidades corporais foi encontrado em $44 \%$ e foi baseado no relato da falta de interesse pela comida $(80 \%)$, no sinal de perda de peso com ingestão adequada de comida (73\%), na aversão ao ato de comer (40\%) e no peso corporal $20 \%$ ou mais abaixo do ideal (40\%). A equipe multidisciplinar deve estar atenta a estes fatores, pois a alimentação é primordial para a promoção, manutenção e/ou recuperação da saúde em todas as fases da vida ${ }^{(28)}$. Os principais fatores de risco que indicaram este diagnóstico foram: fatores biológicos (87\%), psicológicos $(53 \%)$ ou incapacidade de ingerir os alimentos (67\%). O enfermeiro é responsável por identificar as possíveis causas que afetam os pacientes e educá-los, estimulá-los e orientá-los para a adequada ingestão de nutrientes, o que promove a saúde.

Um dos fatores biológicos para a falta de interesse pela comida é que para alimentar-se há um gasto de energia para a metabolização e pacientes com problemas respiratórios dispõem de energia menor, refletindo na perda de apetite. A desnutrição reduz a massa diafragmática e contribui para a diminuição de força e resistência dos músculos respiratórios.

Outro DE potencial encontrado em nosso estudo foi Risco de Infecção (41\%). Estudos semelhantes revelam que o risco de infecção aparece, como um dos mais frequentes diagnósticos de enfermagem identificados ${ }^{(12,23,25)}$. Muitos estudos consideram como fator principal o uso de procedimentos invasivos e em nosso estudo obtivemos o mesmo resultado (100\%), sendo, portanto, o principal fator de risco. Os outros fatores de risco encontrados foram: defesas primárias $(57 \%)$ e secundárias (43\%) inadequadas e desnutrição $(29 \%)$

O DE Dor Aguda foi elaborado a partir das seguintes características definidoras: relato verbal do paciente $(100 \%)$ ou expressões faciais (46\%). Estes dados são semelhantes aos encontrados no estudo realizado com pacientes da clínica-médica ${ }^{(29)}$. Os principais fatores relacionados que compuseram identificar o diagnóstico Dor Aguda encontrados, foram: agentes biológicos (77\%) e físicos.

O DE Fadiga esteve presente em 38\% dos pacientes e está intimamente ligado à privação de sono e troca de gases prejudicada, pois a clientela em estudo verbalizava uma constante falta de energia justificada pelo cansaço $(85 \%)$ por não conseguir dormir em razão da dor ou dispneia e, além disso, por alterações metabólicas advindas da troca de gases prejudicada ou padrão respiratório ineficaz. Os fatores relacionados propiciaram a elaboração deste diagnóstico foram: ansiedade $(77 \%)$, estados de doença $(69 \%)$, depressão $(46 \%)$ e estresse $(31 \%)$. 
Na literatura, a fadiga é conceituada como uma experiência subjetiva de cansaço generalizado, fraqueza, exaustão e falta de energia relacionada ao estresse prolongado advindo ou não de um processo de doença. Porém é um sintoma provém da observação ou verbalização do cliente, da dificuldade de realização das atividades habituais do dia a dia, causando distúrbios físicos e/ou mentais, apresentando redução de sua capacidade de trabalho ${ }^{(30)}$.

A população de idosos que possui alguma doença pulmonar crônica tem dificuldade para manter suas atividades cotidianas pelo decurso da doença ocasionando problemas psicossociais. Desta forma, são necessárias atividades de promoção e prevenção desses problemas, proporcionando a melhora na qualidade de

\section{REFERÊNCIAS}

1. Secretaria Executiva do Ministério da Saúde. Caderno de Informações de Saúde. Informações Gerais. Município: São Paulo. [citado 2008 Jun 19]. Disponível em: http:// tabnet.datasus.gov.br/tabdata/cadernos/SP/ SP_Sao_Paulo_Geral.xls.

2. Toyoshima MT, Ito GM, Gouveia N. Morbidade por doenças respiratórias em pacientes hospitalizados em São Paulo/SP. Rev Assoc Med Bras (1992). 2005;51(4):209-13.

3. Duchiade MP. Poluição do ar e doenças respiratórias: uma revisão. Cad Saúde Pública. 1992;8(3):311-30.

4. Gouveia N, Freitas CU, Martins LC, Marcilio IO. Hospitalizações por causas respiratórias e cardiovasculares associadas à contaminação atmosférica no Município de São Paulo, Brasil. Cad Saúde Pública. 2006;22(12):266977.

5. Silva LC. Compêndio de pneumologia. 2 a ed.São Paulo: Fundo Editorial BYK; 1991.

6. Campedelli MC, Benko MA, Castilho V, Castellanos BE, Gaidzinski RR, Kimura M. Processo de enfermagem na prática. 2a ed. São Paulo: Ática; 2000.

7. Horta WA. Processo de enfermagem. São Paulo: Editora Pedagógica e Universitária: Editora da Universidade de São Paulo; c1979.

8. Gouveia HG, Lopes MH. Diagnósticos de enfermagem e problemas colaborativos mais comuns na gestação de risco. Rev Latinoam Enferm. 2004;12(2):175-82.

9. Potter PA, Perry AG. Fundamentos de enfermagem. $6^{a}$ ed. Rio de Janeiro: Elsevier; 2005.

10. Bettencourt AR, Martins I, Leite AL, Zeitoun SS. Exame do tórax: aparelho respiratório. In: Barros AL, organizadora. Anamnese e exame físico: avaliação diagnóstica de enfermagem no adulto. 2a ed. Porto Alegre: Artmed; 2010. p. 203-33.

11. Bettencourt AR, Leite AL. Propedêutica. In: Barbosa DA, Vianna LA, organizadoras. Enfermagem ambulatorial e hospitalar. Barueri: Manole; 2009. p. 93-104.

12. Volpato MP, Cruz DA. Diagnósticos de enfermagem de pacientes internadas em unidade médico-cirúrgica. Acta Paul Enferm. 2007;20(2):119-24.

13. César CL, Carandina L, Barros MB, Golgbaum M, Porto MC. Inquérito de Saúde no Município de São Paulo 2003 ISA Capital. [citado 2008 Jun 19] Disponível em: http://www.prefeitura.sp.gov.br/cidade/secretarias/ saude/informacoes_em_saude/morbidade/ index.php? $\mathrm{p}=6168$ vida destes idosos e a diminuição das complicações de doenças pulmonares ${ }^{(31)}$.

\section{CONCLUSÃO}

Os diagnósticos de enfermagem que apresentaram frequência de 38\% ou mais foram: Troca de Gases Prejudicada, Privação do Sono, Risco para Quedas, Nutrição Desequilibrada menor do que as necessidades corporais, Risco de Infecção, Dor Aguda e Fadiga. Estudos epidemiológicos que informam sobre a frequência dos diagnósticos de enfermagem em uma população contribuem para organizar a base de conhecimento de enfermagem e prever os cuidados de enfermagem necessários a uma população específica.

14. North American Nursing Diagnosis Association. Diagnósticos de enfermagem da NANDA: definições e classificação 2007-2008. Porto Alegre: Artmed; 2008.

15. Oliva AP, Lopes DA, Volpato MP, Hayashi AA. Atitudes de alunos e enfermeiros frente ao diagnóstico de enfermagem. Acta Paul Enferm. 2005;18(4):361-7.

16. Zamboni M, Pereira CA. Pneumologia: diagnóstico e tratamento. São Paulo: Atheneu; 2006.

17. Brasil. Ministério da Saúde. Secretaria de Atenção à Saúde. Instituto Nacional de Câncer. Estimativas da incidência e mortalidade por câncer no Brasil. Rio de Janeiro: INCA; 2006.

18. Cardoso AP, Noronha A, Nakatani J, Viana LG, Dalcolmo MP. I Consenso brasileiro sobre pneumonias. Pneumonias adquiridas na comunidade. J Pneumol. 1998;24(2):66-72.

19. Martins I, Gutiérrez MG, Barros AL. Identificação e validação das características definidoras do diagnóstico de enfermagem: limpeza ineficaz de vias aéreas. Acta Paul Enferm. 1999;12(1):34-42.

20. Martins I, Gutiérrez MG. Intervenções de enfermagem para o diagnóstico de enfermagem Desobstrução ineficaz de vias aéreas. Acta Paul Enferm. 2005;18(2):143-9.

21. Monteiro FP, Silva VM, Lopes MVO, Araújo TL. Condutas de enfermagem para o cuidado à criança com infecção respiratória: validação de um guia. Acta Paul Enferm. 2007;20(4):458-63.

22. Siskind MM. A standart of care for nursing diagnoses of ineffective airway clearence. Heart Lung. 1989;18(5):477-82.

23. Silva MR, Bettencourt AR, Diccini S, Belasco A, Barbosa DA. Diagnósticos de enfermagem em portadores da Síndrome da Imunodeficiência Adquirida. Rev Bras Enferm. 2009;62(1):92-9.

24 Zeitoun SS, de Barros AL, Michel JL, de Bettencourt AR. Clinical validation of the signs and symptoms and the nature of the respiratory nursing diagnoses in patients under invasive mechanical ventilation. J Clin Nurs. 2007;16(8):1417-26.

25. Jesus CA, Carvalho EC. Diagnóstico de enfermagem em clientes com alterações hematológicas: uso da Taxonomia I da NANDA. Rev Latinoam Enferm. 1997;5(4):91-9.

26. Lima MA, Trad LA. A dor crônica sob o olhar médico: modelo biomédico e prática clínica. Cad Saúde Pública $=$ Rep Public Health. 2007;23(11):2672-80.

27. Araújo LA, Bachion MM. Diagnósticos de Enfermagem do Padrão Mover em idosos de uma comunidade atendida 
pelo Programa Saúde da Família. Rev Esc Enferm USP. 2005;39(1):53-61.

28. Ferreira IM. Doença pulmonar obstrutiva crônica e desnutrição: por que não estamos vencendo a batalha? J Pneumol. 2003;29(2):107-15.

29. Fontes CM, Cruz DA. Diagnóstico de enfermagem documentados para pacientes de clínica médica. Rev Esc
Enferm USP. 2007;41(3):395-402.

30. Menezes MF, Camargo TC. A fadiga relacionada ao câncer como temática na enfermagem oncológica. Rev Latinoam Enferm. 2006;14(3):442-7.

31. Francisco PM, Donalisio MR, Barros MB, César CL, Carandina L, Goldbaum M. Fatores associados à doença pulmonar em idosos. Rev Saúde Pública. 2006;40(3):428-35. 